\title{
Sampling sites for detection of feline herpesvirus-1, feline calicivirus and Chlamydia felis in cats with feline upper respiratory tract disease
}

Journal of Feline Medicine and Surgery 2015, Vol. 17(12) 1012-1019 (C) ISFM and AAFP 2015

Reprints and permissions: sagepub.co.uk/journalsPermissions.nav DOI: $10.1177 / 1098612 \times 15569615$ jfms.com

(S)AGE

\author{
Catharina Schulz ${ }^{1}$, Katrin Hartmann ${ }^{1}$, Ralf S Mueller ${ }^{1}$, \\ Chris Helps ${ }^{2}$ and Bianka S Schulz ${ }^{1}$
}

\begin{abstract}
Objectives Feline herpesvirus-1 (FHV-1), feline calicivirus (FCV) and Chlamydia felis are involved in feline upper respiratory tract disease (FURTD). Clinical signs caused by these agents can overlap, and the involvement of certain pathogens is often unpredictable. The objectives of this study were to compare detection rates of FHV-1, FCV and $C$ felis at different sampling sites, and to investigate the correlation between positive test results and clinical signs in cats with FURTD.

Methods Swabs were taken from the nose, pharynx, tongue and conjunctiva of 104 cats with signs of FURTD. Real-time PCR was performed on all samples for the detection of FHV-1, FCV and C felis.

Results Infectious agents were identified in 93 (89.4\%) cats. Of these, 55.8\% were positive for $\mathrm{FHV}-1,50.0 \%$ for FCV and $35.6 \%$ for $C$ felis. FCV was found more frequently in the oropharynx (92.3\% of FCV-positive cats) and on the tongue $(90.4 \%)$ than the conjunctiva $(38.5 \%)(P<0.001)$. There was no significant difference between the four sampling sites for the detection of FHV-1 and $C$ felis. If nasal samples had also been taken, $94.9 \%$ of FHV-1-positive cats, $96.2 \%$ of FCV-positive cats and $81.1 \%$ of $C$ felis-positive cats would have been detected.

Conclusions and relevance The oropharynx can be recommended as the preferred single sampling site for the detection of FCV, FHV-1 and C felis if only one sample can be taken; however, taking samples at different sites significantly increases the detection rate for all pathogens studied. Interestingly, sampling from a site with FURTDassociated lesions did not increase the likelihood of detecting the infectious agents.
\end{abstract}

Accepted: 17 December 2014

\section{Introduction}

Feline upper respiratory tract disease (FURTD) remains a significant problem, despite the widespread use of vaccination over the past 30 years. ${ }^{1}$ It is usually caused by feline calicivirus (FCV), feline herpesvirus (FHV)- $1^{2}$ or Chlamydia felis ${ }^{3}$. Common clinical signs of FURTD include nasal and ocular discharge, sneezing, dyspnoea and coughing. ${ }^{4}$ In addition, oral ulcerations are often observed in cats with FCV infection, ${ }^{5}$ and dendritic corneal ulcers can occur in cats infected with FHV-1.6,7 $C$ felis mainly causes acute or chronic conjunctivitis and blepharospasm with serous or mucopurulent ocular discharge. ${ }^{8-10}$

A presumptive diagnosis of the pathogen(s) involved in FURTD is commonly established based on the presence or absence of certain clinical signs; however, there is considerable overlap in clinical signs between the three pathogens, and mixed infections can occur. ${ }^{11-13}$ As a consequence, the pathogens involved in FURTD can only be definitively identified by laboratory tests, which can be used to guide anti-infective treatment, such as antiviral drugs or antibiotics, and to control the infections in multicat households.

${ }^{1}$ Clinic of Small Animal Medicine, LMU University of Munich, Munich, Germany

2Molecular Diagnostic Unit, Langford Veterinary Services, University of Bristol, Bristol, UK

\section{Corresponding author:}

Catharina Schulz DVM, Clinic of Small Animal Medicine, LMU University of Munich, Veterinaerstr 13, D-80539 Munich, Germany

Email: c.schulz@medizinische-kleintierklinik.de 
PCR for detection of FHV-1 and C felis, and reverse transcriptase PCR (RT-PCR) for detection of FCV are considered rapid, sensitive and inexpensive. ${ }^{14}$ Some studies have shown that PCR has a higher sensitivity than virus isolation and enzyme-linked immunosorbent assays for the detection of FHV-1, FCV and C felis. ${ }^{15-19}$

The aim of this study was to compare four different sampling sites for detection of FHV-1, FCV and C felis by PCR or RT-PCR (FCV) in cats with FURTD in order to identify a preferred sampling site for each pathogen. Furthermore, the correlation between clinical signs and detection site was investigated for the three pathogens.

\section{Material and methods Patients}

In the study, 104 cats with signs of FURTD that were presented to the Clinic of Small Animal Medicine of the LMU University of Munich between July 2012 and October 2013 were included. All cats were included because they were suspected of having FHV-1-, FCV-, or C felis-related disease. These cats consisted of a population of first-opinion and referral cases. Cats were eligible to enter the study if they had at least one clinical sign of FURTD, including upper respiratory or ocular signs. Respiratory signs included nasal discharge, sneezing, and ulceration of the pharynx or tongue. Ocular signs included conjunctivitis, ocular discharge, keratitis and dendritic corneal ulcers. Clinical signs of conjunctivitis were defined as hyperaemia, chemosis, epiphora or ocular discharge. Both treated (antibiotics, antiinflammatories, mucolytic therapy, pain medication, L-lysine) and vaccinated cats were included, as were cats with acute, as well as chronic, disease. Information regarding breed, age, sex, vaccination status, housing, duration of clinical signs, additional diseases and current therapy were documented using a standardised protocol for each cat. A general physical examination and specific examination of the respiratory tract were performed on each cat. All parameters were documented in a standardised questionnaire. A clinical scoring system was used that evaluated clinical parameters on a scale of 0 (absent) to 3 (severe). The subdivision of the cats into the groups 'mildly', 'moderately' and 'severely' affected was performed based on a scoring system that was previously published in an earlier study. ${ }^{20}$ A complete ophthalmic examination was performed in 6/7 (85.7\%) cats with keratitis.

\section{Sample technique}

Within $48 \mathrm{~h}$ of admission, four dry cotton-tipped swabs per cat were collected, one each from the nasal cavity, the conjunctiva, the tongue and the oropharynx. Sampling was always performed on the side showing more severe clinical signs. If there was no difference between both sides, the left side was sampled. Nasal swabs were obtained by gently rolling the sterile swab in the anterior aspect of the nares after removing any excess mucous. Pharyngeal swabs were obtained by gently rotating the swab in the caudal oropharynx, trying to avoid contact with the tongue. The conjunctival swab was rolled along the ventral conjunctival fornix. No ocular anaesthetic was used when collecting the conjunctival swabs. The anterior tip of the tongue was sampled by rolling the swab on the mucosa. Samples were stored at $-20^{\circ} \mathrm{C}$ until analyses were performed.

\section{PCR}

For isolation of total nucleic acid (DNA and RNA), the Nucleospin Blood kit (Macherey Nagel) was used. Cotton swabs were placed in a solution of $200 \mu \mathrm{l}$ phosphate-buffered saline (Carl Roth), $200 \mu$ l buffer BQ1 and $20 \mu$ proteinase $\mathrm{K}$. Swabs were incubated at $70^{\circ} \mathrm{C}$ for 15 mins with shaking at $700 \mathrm{rpm}$ (Vortemp 56; Labnet), after which the manufacturer's protocol was followed. Total nucleic acid was eluted with $100 \mu \mathrm{l}$ of biotin elution buffer and stored at $-80^{\circ} \mathrm{C}$.

A quantitative PCR (qPCR) for FHV-1 and a qRT-PCR for FCV were performed as described previously ${ }^{21}$ both included internal amplification controls. A qPCR to detect $C$ felis and feline 28S rDNA (endogenous internal control) was set up as follows: $12.5 \mu \mathrm{l}$ of $2 \times$ GoTaq PCR Master Mix (Promega), $200 \mathrm{nM}$ each of 28S rDNA forward and reverse primers, $200 \mathrm{nM}$ each of $C$ felis forward and reverse primers, $50 \mathrm{nM} 28 \mathrm{~S}$ rDNA Texas Red-BHQ2 probe, $50 \mathrm{nM} \mathrm{C}$ felis FAM-BHQ1 probe (sequences described previously ${ }^{22}$ ), $4.5 \mathrm{mM} \mathrm{MgCl}$ final concentration, $5 \mu \mathrm{l}$ genomic DNA and water to $25 \mu \mathrm{l}$. The reaction was run in an Agilent MX3005P and incubated at $95^{\circ} \mathrm{C}$ for 2 mins followed by 45 cycles of $15 \mathrm{~s}$ at $95^{\circ} \mathrm{C}$ and $30 \mathrm{~s}$ at $60^{\circ} \mathrm{C}$. Fluorescence was detected at $520 \mathrm{~nm}$ and 610 $\mathrm{nm}$ at each annealing step $\left(60^{\circ} \mathrm{C}\right)$.

All qPCR data were analysed using the Agilent MX3005P software.

\section{Statistical evaluation}

For statistical analysis, GraphPad Prism was used. Fisher's exact test was used for all comparisons. At first, a global $P$ value was determined $(2 \times 4$ contingency table), followed by post-hoc analysis if a global significant difference between all four sampling sites was detected. Two sampling sites were compared in $2 \times 2$ cross tables. The level of significance was set at $P<0.05$ for these comparisons. An adjustment with a Bonferroni correction was used for the comparison of all four sampling sites (giving a total of six comparisons), leading to a level of significance of $P<0.008$.

\section{Results}

Signalment and clinical signs

The study included 94 domestic shorthair cats, one domestic longhair and nine purebred cats (five 
Table 1 Detection rates of feline herpesvirus (FHV)-1, feline calicivirus (FCV) and Chlamydia felis in cats with clinical signs of feline upper respiratory tract disease $(n=104)$ at different sampling sites (pharynx, tongue, nose and conjunctiva) and number of positive swabs (total number of swabs $=416$ )

\begin{tabular}{lccl} 
& FHV-1 & FCV & C felis \\
\hline Total detection rate & $58(55.8)$ & $52(50.0)$ & $37(35.6)$ \\
Total number of positive swabs & $164(39.4)$ & $151(36.3)$ & $89(21.4)$ \\
Sampling sites & & & \\
Pharynx [95\% Cl] & $40(69.0)[56.2-79.4]$ & $48(92.3)[81.8-97.0]^{*}$ & $25(67.6)[51.5-80.4]$ \\
Tongue [95\% Cl] & $41(70.7)[57.9-80.8]$ & $47(90.4)[79.4-95.8]^{\dagger}$ & $17(45.9)[31.0-61.6]$ \\
Nose [95\% Cl] & $47(81.0)[69.1-89.1]$ & $36(69.2)[55.7-80.0]$ & $22(59.5)[43.5-73.7]$ \\
Conjunctiva [95\% Cl] & $36(62.0)[49.2-73.4]$ & $20(38.5)[26.5-52.0]^{*}$ & $25(67.6)[51.5-80.4]$ \\
Pvalue & $>0.05$ & $<0.0001^{*}$ & $>0.05$ \\
& & $<0.0001^{+}$ & \\
& & Other comparisons: $>0.05$ &
\end{tabular}

Values are given as $\mathrm{n}(\%)$

${ }^{*}$ Comparison between detection rates of FCV in pharynx and conjunctiva tComparison between detection rates of FCV in tongue and conjunctiva $\mathrm{Cl}=$ confidence interval

Siamese, two Maine Coon, one British Shorthair, one Persian). Thirty-two $(30.8 \%)$ cats were female intact, $17(16.3 \%)$ were female spayed, $29(27.8 \%)$ were male intact and $26(25.1 \%)$ were male neutered. The age of the cats was between 4 weeks and 19 years (median 6 months).

Of all 104 cats, $35(34.0 \%)$ lived in public or private animal shelters, $21(20.2 \%)$ in multi-cat colonies on farms and $40(38.8 \%)$ were client-owned. Eight cats $(7.7 \%)$ were strays. Most cats $(86.4 \%)$ lived in multi-cat households. Fourteen of 104 cats had been treated with oxytetracycline eye ointment at the time of presentation, one cat with oral doxycycline, 10 with subcutaneous enrofloxacin and two with oral pradofloxacin.

Most cats showed more than one clinical sign. Eightysix $(82.7 \%)$ cats showed nasal discharge, $85(81.7 \%)$ showed sneezing, $83(79.8 \%)$ showed ocular discharge, $16(15.4 \%)$ showed oral ulcerations, $56(53.8 \%)$ showed conjunctivitis and seven $(6.7 \%)$ showed keratitis. Further clinical abnormalities included lethargy $(68 / 104 ; 65.4 \%)$, increased body temperature $(13 / 104 ; 12.5 \%)$, reduced appetite $(32 / 104 ; 30.8 \%)$, anorexia $(10 / 104 ; 9.6 \%)$, tachypnoea $(26 / 104 ; 25.0 \%)$, salivation $(14 / 104 ; 13.5 \%)$ and gingivostomatitis $(20 / 104 ; 19.2 \%)$. Lameness due to polyarthritis was observed in three $(2.9 \%)$ cats. Clinical signs were assessed as moderate in $22.3 \%$ and as severe in $27.1 \%$ of cats. Fifty-seven (55.0\%) cats had to be hospitalised. The duration of clinical signs was unknown for 35 (33.9\%) cats; 37 (35.9\%) had shown signs between 1 and 21 days, and $31(30.0 \%)$ cats had signs of FURTD for more than 21 days.

\section{Detection rates of FHV-1, FCV and C felis}

Overall, 416 samples from four different sampling sites were available from 104 cats with FURTD. At least one pathogen was detected in $93(89.4 \%)$ cats. FHV-1 was detected in $58(55.8 \%), \mathrm{FCV}$ in $52(50.0 \%)$ and $C$ felis in $37(35.6 \%)$ cats. Detection rates of the three pathogens at the four different sampling sites are displayed in Table 1. There was no significant difference in the detection of FHV-1 and C felis between the four sampling sites. For FCV, a global difference was detected between the four sampling sites $(P<0.001)$, with the virus being detected significantly more often from the oropharynx and from the tongue than from the conjunctiva $(P<0.0001)$.

Forty-six of $104(46.2 \%)$ cats were infected with a single pathogen, with the remaining $47(44.2 \%)$ pathogeninfected cats having multiple-pathogen infection. Infections with two pathogens were detected in 40/104 $(37.5 \%)$ cats. The ratio of single-pathogen to multiplepathogen infection was 28:30 for FHV-1, 10:42 for FCV and 8:29 for $C$ felis. Table 2 shows the detection rates for multiple-pathogen infections at the different sampling sites. Co-infections of FCV and C felis were detected significantly more often in the pharynx than in the conjunctiva $(P=0.001)$. There was no difference between the sampling sites for the other multiple-pathogen infections.

Table 3 shows the number of locations for detection of the three pathogens. By taking only pharyngeal swabs for detection of the three pathogens, 18/58 (31.0\%) FHV1-positive cats would not have been identified, and $4 / 52$ $(7.7 \%)$ FCV-positive and $12 / 37(32.4 \%)$ C felis-positive cats would have been missed.

Table 4 demonstrates single- or multiple-pathogen detection rates in cats with oral ulceration, conjunctivitis or keratitis. Results for cats infected with a single pathogen showing oral ulceration, conjunctivitis or keratitis are displayed in Table 5. 
Table 2 Detection rates for multiple-pathogen infections in cats with clinical signs of feline upper respiratory tract disease at four different sampling sites (pharynx, nose, tongue and conjunctiva)

\begin{tabular}{|c|c|c|c|}
\hline Total detection rate & $18(17.3)$ & $17(16.3)$ & $5(4.8)$ \\
\hline Sampling sites & FHV-1 + FCV & $\mathrm{FCV}+\mathrm{C}$ felis & FHV-1 + C felis \\
\hline Pharynx [95\% Cl] & $8(44.4)$ [24.6-66.3] & $13(76.5)[52.7-90.4]^{*}$ & 1 (20.0) [3.6-62.5] \\
\hline Tongue $[95 \% \mathrm{Cl}]$ & 9 (50.0) [29.0-71.0] & $10(58.8)[36.0-78.3]$ & 1 (20.0) [3.6-62.5] \\
\hline Nose $[95 \% \mathrm{Cl}]$ & 8 (44.4) [24.6-66.3] & $8(47.0)[26.2-69.0]$ & $2(40.0)[11.8-76.9]$ \\
\hline Conjunctiva [95\% Cl] & 4 (22.2) [9.0-45.2] & $4(23.5)[9.6-47.3]^{*}$ & 2 (40.0) [11.9-76.9] \\
\hline$P$ value $[95 \% \mathrm{Cl}]$ & $>0.05$ & $\begin{array}{l}0.001^{*} \\
\text { Other comparisons } \\
>0.05\end{array}$ & $>0.05$ \\
\hline
\end{tabular}

Values given as $\mathrm{n}(\%)$

${ }^{*}$ Comparison between detection rates of FCV and $C$ felis multiple infection in pharynx and conjunctiva

FHV-1 = feline herpesvirus- $1 ; \mathrm{Cl}=$ confidence interval; FCV = feline calicivirus; $C$ felis = Chlamydia felis

Table 3 Detection rates of feline herpesvirus (FHV)-1, feline calicivirus (FCV) and Chlamydia felis for one, two, three and four locations in cats with respiratory tract disease

\begin{tabular}{|lccc|}
\hline Number of locations & FHV-1 $(n=58)$ & FCV $(n=52)$ & C felis $(n=37)$ \\
\hline 1 & $11(19.0)$ & $5(9.6)$ & $18(48.6)$ \\
2 & $12(20.7)$ & $13(26.9)$ & $1(2.7)$ \\
3 & $11(18.7)$ & $16(30.8)$ & $3(8.1)$ \\
4 & $24(41.4)$ & $18(34.6)$ & $15(40.5)$ \\
\hline
\end{tabular}

Values are given as $n(\%)$

Table 4 Detection rates of feline herpesvirus (FHV)-1, feline calicivirus (FCV) and Chlamydia felis in cats infected with single or multiple pathogens, with clinical signs of oral ulcerations, conjunctivitis, keratitis or rhinitis

\begin{tabular}{lllllc} 
Clinical signs & $\begin{array}{l}\text { Total detection } \\
\text { rates }\end{array}$ & FHV-1 & FCV & C felis & $P$ value \\
\hline $\begin{array}{l}\text { Oral ulcerations } \\
\text { [95\% Cl] }\end{array}$ & $16(15.4)$ & $11(68.7)[44.4-85.8]$ & $\begin{array}{l}13(81.2)^{\star} \\
{[56.0-93.4]}\end{array}$ & $\begin{array}{l}5(31.2)^{*} \\
{[14.2-55.6]}\end{array}$ & $0.001^{*}$ \\
Conjunctivitis & $56(53.8)$ & $30(53.6)[40.7-66.0]$ & $30(53.6)$ & $28(50.0)$ & $>0.05$ \\
{$[95 \% \mathrm{Cl}]$} & & & {$[40.7-66.0]$} & {$[37.3-62.7]$} & \\
Keratitis [95\% Cl] & $7(6.7)$ & $5(71.4)[35.9-91.8]$ & $0(0)[0-35.4]$ & $2(28.6)[8.2-64.1]$ & $>0.05$ \\
Rhinitis [95\% Cl] & $61(58.7)$ & $35(57.4)[44.9-68.9]^{\dagger}$ & $30(49.2)$ & $21(34.4)^{\dagger}$ & $0.01^{\dagger}$ \\
& & & {$[37.1-61.4]$} & {$[23.8-47.0]$} &
\end{tabular}

Values are given as $\mathrm{n}(\%)$

${ }^{*}$ Comparison in cats with oral ulcerations between FCV infection and $C$ felis infection

${ }^{+}$Comparison in cats with rhinitis between FHV-1 infection and $C$ felis infection

$\mathrm{Cl}=$ confidence interval

\section{Discussion}

The aim of this study was to determine preferred sampling sites for the detection of FHV-1, FCV and C felis in cats with FURTD. Several previous studies have investigated the detection rates of these pathogens in cats with FURTD; however, this is the first study to compare data from four different sampling sites.

In this study there was no difference in the detection rates of FHV-1 between the different sampling sites. FHV-1 tended to be most frequently detected in the nose, but this difference was not significant compared with other locations. This is in concordance with results of a previous study, in which the detection rates of FHV-1 in cats with FURTD obtained by pharyngeal and nasal swabs were almost identical. ${ }^{23}$ This suggests that the pharynx and the nasal cavity are suitable sampling sites for the detection of FHV-1. However, while performing the current study it became obvious that nasal swabs were much more difficult to take than pharyngeal swabs, something that has previously been reported. ${ }^{23}$ 
Table 5 Detection rates of feline herpesvirus (FHV)-1, feline calicivirus (FCV) and Chlamydia felis in cats infected with single pathogens, with clinical signs of oral ulcerations, conjunctivitis, keratitis or rhinitis

\begin{tabular}{|c|c|c|c|c|c|}
\hline Clinical signs & $\begin{array}{l}\text { Total } \\
\text { detection } \\
\text { rates }\end{array}$ & $\mathrm{FHV}-1$ & FCV & C felis & $P$ value \\
\hline $\begin{array}{l}\text { Oral ulcerations } \\
{[95 \% \mathrm{Cl}]}\end{array}$ & $5(4.8)$ & 1 (20.0) [3.6-62.5] & $3(60.0)$ [23.1-88.2] & 1 (20.0) [3.6-62.5] & $>0.05$ \\
\hline $\begin{array}{l}\text { Conjunctivitis } \\
\text { [95\% Cl] }\end{array}$ & $18(17.3)$ & $12(66.6)[43.8-83.7]^{\star}$ & $0(0)[0-17.6]^{*}$ & 6 (33.3) [16.3-56.3] & $\begin{array}{l}<0.0001^{*} \\
\text { Other comparisons } \\
>0.05\end{array}$ \\
\hline $\begin{array}{l}\text { Keratitis } \\
{[95 \% \mathrm{Cl}]}\end{array}$ & $5(4.8)$ & $3(60.0)$ [23.1-88.2] & $0(0)[0-43.5]$ & $2(40.0)[11.8-76.9]$ & $>0.05$ \\
\hline $\begin{array}{l}\text { Rhinitis } \\
{[95 \% \mathrm{Cl}]}\end{array}$ & $26(25.0)$ & $17(65.4)[46.2-80.6]^{\ddagger \ddagger}$ & $3(11.5)[4.0-29.0]^{\dagger}$ & $6(23.0)[11.0-42.1]^{\ddagger}$ & $\begin{array}{l}<0.0001^{\dagger} 0.004^{\ddagger} \\
\text { Other } \\
\text { comparisons } \\
>0.05\end{array}$ \\
\hline
\end{tabular}

Values are given as $\mathrm{n}(\%)$

${ }^{*}$ Comparison in cats with conjunctivitis between FHV-1 infection and FCV infection

tComparison in cats with rhinitis between FHV-1 infection and FCV infection

$\neq$ Comparison in cats with rhinitis between FHV-1 infection and $C$ felis infection

$\mathrm{Cl}=$ confidence interval

In this study, FCV was detected significantly more often on the pharynx and tongue compared with other locations. This was expected as a previous study had shown that in six cats experimentally infected with FCV the virus was isolated more frequently from pharyngeal swabs than from nasal and conjunctival swabs. ${ }^{24}$ Two previous studies of cats with FURTD also demonstrated that more pharyngeal than conjunctival swabs were positive for FCV, 25,26 while in another study in cats with FURTD, there was no difference between conjunctival and pharyngeal swabs. ${ }^{14}$ However, the latter study only had a $9.6 \%$ detection rate for $\mathrm{FCV}^{14}$ In contrast, in a report from Germany investigating 68 cats with FURTD, FCV was detected in $35.8 \%$ of pharyngeal swabs and in $44.0 \%$ of conjunctival swabs. ${ }^{27}$ Most of these studies confirm the results of the present study, indicating that pharyngeal swabs, as well as swabs from the tongue, can be recommended as sampling sites for the diagnosis of FCV infection; however, 4/52 FCV-positive cats would have been missed by taking swabs only from the pharynx, and five FCV-positive cats would not have been detected by taking swabs only from the tongue. It was an unexpected finding of the present study that there was no difference between the four sampling sites with regard to the detection rate of $C$ felis, as this pathogen was found in the oropharynx and conjunctiva with equal frequency. Chlamydia felis has been described predominantly as a conjunctival pathogen in cats. ${ }^{9,10}$ Pharyngeal shedding of the organism has been previously described in cats with FURTD; ${ }^{28}$ however, in contrast to results of the present study, detection of $C$ felis has mostly been shown from conjunctival swabs..$^{25,29}$

The most common infectious agent detected in this study was FHV-1. Compared with previous studies, a much higher prevalence of FHV-1 (55.8\%), FCV (50.0\%) and $C$ felis (35.6\%) was obtained. ${ }^{30-32}$ One explanation for this is the fact that four samples from different sites were collected from each animal, while previous studies established prevalence data based upon samples taken from one or two sites. ${ }^{14,23,30}$ It is also likely that the higher prevalence rates in the present study were owing to the selection of the cats showing signs of FURTD, with $>50 \%$ of the cats showing moderate or severe clinical signs and requiring hospitalisation. Other studies have shown that the detection rates of FHV-1, FCV and C felis can vary depending on the severity of disease. ${ }^{33,34}$ In addition, $86.4 \%$ of cats in the present study originated from multicat households or animal shelters, and both crowding and hygiene status can influence the prevalence rates.22

Dual or multiple infections with FHV-1, FCV and C felis were detected in half of the pathogen-positive cats, indicating that infections with more than one pathogen are very common in cats with FURTD. In previous studies, cats with $C$ felis infection were commonly co-infected with FHV-1 or with FCV, 14,35,36, while in the present study $C$ felis infection was mostly accompanied by FCV infection. Furthermore, co-infections with FHV-1 and FCV were also commonly detected. Co-infection with FCV and $C$ felis was detected more frequently in the pharynx; however, by sampling only this region, shedding of pathogens in other sampling sites would have been missed. Therefore, sampling multiple sites can be recommended for cats with single- and multiple-pathogen infections.

In cats with rhinitis, FHV-1 was detected more frequently than FCV and $C$ felis, which agrees with results of previous investigations. . $0,37^{3}$ Similar to previous studies, ${ }^{38-41} \mathrm{FCV}$ tended to be detected more frequently than 
other pathogens in cats with oral ulcerations, and FHV-1 tended to be the most prevalent pathogen in cats with keratitis; ${ }^{38-40}$ however, both differences were not statistically significant. Nevertheless, the small number of patients with oral ulcerations and keratitis has to be considered. In cats with conjunctivitis, FHV-1 was identified significantly more often than FCV, which is consistent with findings in other studies. ${ }^{22,35} \mathrm{C}$ felis was detected as a single pathogen in six cats with rhinitis that did not show signs of conjunctivitis, indicating that $C$ felis might be able to cause respiratory signs and not just conjunctivitis. In experimental studies, nasal discharge and sneezing has been observed in cats after infection with $C$ felis. $9,10,42$ The low detection rate of $C$ felis in cats with conjunctivitis might have been influenced by previous treatment with antibiotics. Therefore, it might be possible that $C$ felis, even though the causative agent for conjunctivitis, was not shed at the time of sampling in some cats.

The fact that half of the cats were infected with more than one pathogen confounds interpretation of the clinical signs as they cannot be clearly attributed to a certain pathogen. When cats infected with a single pathogen were investigated (Table 5), conjunctivitis was associated with FHV-1-infection, although numbers of cats were very low; however, this could not be detected when clinical signs were evaluated in all cats infected with one or more pathogens (Table 4). It cannot be excluded that some cats were shedding infectious organisms, while their clinical signs were caused by other non-infectious problems such as nasal neoplasia and chronic rhinosinusitis, as cats with a chronic course of disease were also included in the study. The qPCR can only detect the presence of the organism, but cannot predict a relationship between infection status and clinical signs. To assess the relationship between infection with a certain organism and clinical disease, infection studies would be needed in an experimental setting.

While some cats were shedding FHV-1, FCV and $C$ felis only in one location, almost equal numbers of cats were positive for one of the pathogens in two, three or four sampling sites. By taking samples from only the pharynx, $31.0 \%$ of FHV-1-positive, 7.7\% of FCV-positive and $32.4 \%$ of $C$ felis-positive cats would have been missed. If nasal samples had also been taken, $94.9 \%$ of FHV-1positive cats, $96.2 \%$ of FCV-positive cats and $81.1 \%$ of $C$ felis-positive cats would have been detected. By taking pharyngeal and conjunctival swabs, $89.2 \%$ of $C$ felispositive cats would have been detected. Therefore, it can be recommended to take a minimum of pharyngeal and nasal samples to increase the detection rates of these three pathogens.

The number of cats with keratitis and pharyngeal ulceration was relatively small; hence, it is not possible to make recommendations for preferred sampling sites.
Surprisingly, the detection rate of $C$ felis in cats with conjunctivitis tended to be higher in the pharynx and nose than in the conjunctiva, but this difference was not significant, and the timing of sampling might have influenced detection. In an experimental study with 26 $C$ felis-infected cats, the organism was isolated from the conjunctiva of all infected cats 3 days after inoculation and from the nose of all infected cats five days after inoculation. ${ }^{9}$ Therefore, sampling at different time points post-infection could result in different $C$ felis detection rates at different sampling sites, although, to our knowledge, the temporal shedding of $C$ felis in the oropharynx has not been investigated. In cats with nasal discharge, ocular discharge and sneezing, all single- or multiple-pathogen infections were detected more frequently in the pharynx than in the conjunctiva.

One limitation of the present study was the high number of patients with multiple infections, making associations between detection of a single pathogen and clinical signs difficult. Another limitation was the low number of patients with specific disease conditions, such as oral ulcerations and keratitis, which have been linked to infections with specific pathogens. Furthermore, detection of the pathogens might have been influenced by the fact that cats with acute, as well as chronic, illness were included in the study, and duration of illness was unknown in one-third of cats. In addition, some cats were pre-treated with systemic or local antimicrobials, which could have influenced pathogen detection rates in these cats.

\section{Conclusions}

The results obtained in this study have important clinical implications for veterinary practice. Data suggest that the pharynx should be the preferred sampling site for the detection of FHV-1, FCV and C felis in cats with FURTD, if only one sample can be taken. However, obtaining additional samples from other locations significantly increases the chance of detection for all three pathogens. Selection of the sampling site for detection of the three pathogens cannot be based on clinical signs alone.

Acknowledgements We thank the technicians at Langford Veterinary Services for processing the swabs and performing the quantitative PCRs and quantitative reverse transcriptase PCRs. We thank Dr Sven Reese for advice on statistical analysis.

Conflict of interest The authors do not have any potential conflicts of interest to declare.

Funding We thank the Clinic of Small Animal Medicine of the LMU University of Munich for providing financial support for the study. 


\section{References}

1 Radford AD, Gaskell RM and Dawson S. Feline viral upper respiratory disease. In: King LG (ed). Textbook of respiratory disease in dogs and cats. Missouri: WB Saunders, 2004, pp 271-283.

2 Gaskell RM. Upper respiratory disease in the cat (including chlamydia): control and prevention. Feline Pract 1993; 21: 29-34.

3 Sykes JE. Feline upper respiratory tract pathogens: herpesvirus-1 and calicivirus. Compend Contin Educ Pract Vet 2001; 23: 166-177.

4 Hoover EA, Rohovsky MW and Griesemer RA. Experimental feline viral rhinotracheitis in the germfree cat. $A m J$ Pathol 1970; 58: 269-282.

5 Flagstad A. Experimental picornavirus infection in cats. Acta Vet Scand 1973; 14: 501-510.

6 Andrew SE. Ocular manifestations of feline herpesvirus. J Feline Med Surg 2001; 3: 9-16.

7 Roberts SR, Dawson CR, Coleman V, et al. Dendritic keratitis in a cat. J Am Vet Med Assoc 1972; 161: 285-289.

8 Wills JM, Gruffydd-Jones TJ, Richmond SJ, et al. Effect of vaccination on feline Chlamydia psittaci infection. Infect Immun 1987; 55: 2653-2657.

9 Masubuchi K, Nosaka H, Iwamoto K, et al. Experimental infection of cats with Chlamydophila felis. J Vet Med Sci 2002; 64: 1165-1168.

10 Hoover EA, Kahn DE and Langloss JM. Experimentally induced feline chlamydial infection (feline pneumonitis). Am J Vet Res 1978; 39: 541-547.

11 Sykes JE, Browning GF, Anderson G, et al. Differential sensitivity of culture and the polymerase chain reaction for detection of feline herpesvirus 1 in vaccinated and unvaccinated cats. Arch Virol 1997; 142: 65-74.

12 Cello RM. Clues to differential diagnosis of feline respiratory infections. J Am Vet Med Assoc 1971; 158: 968-973.

13 [No authors listed] Report of the panel of the colloquium on selected feline infectious diseases. J Am Vet Med Assoc 1970; 157: 2043-2051.

14 Sykes JE, Allen JL, Studdert VP, et al. Detection of feline calicivirus, feline herpesvirus 1 and Chlamydia psittaci mucosal swabs by multiplex RT-PCR/PCR. Vet Microbiol 2001; 81: 95-108.

15 Burgesser KM, Hotaling S, Schiebel A, et al. Comparison of PCR, virus isolation, and indirect fluorescent antibody staining in the detection of naturally occurring feline herpesvirus infections. J Vet Diagn Invest 1999; 11: 122-126.

16 Rampazzo A, Appino S, Pregel P, et al. Prevalence of Chlamydophila felis and feline herpesvirus 1 in cats with conjunctivitis in northern Italy. J Vet Intern Med 2003; 17: 799-807.

17 Reubel GH, Ramos RA, Hickman MA, et al. Detection of active and latent feline herpesvirus 1 infections using the polymerase chain reaction. Arch Virol 1993; 132: 409-420.

18 Stiles J, McDermott M, Willis M, et al. Comparison of nested polymerase chain reaction, virus isolation, and fluorescent antibody testing for identifying feline herpesvirus in cats with conjunctivitis. Am J Vet Res 1997; 58: 804-807.

19 Stiles J, McDermott M, Bigsby D, et al. Use of nested polymerase chain reaction to identify feline herpesvirus in ocular tissue from clinically normal cats and cats with corneal sequestra or conjunctivitis. Am J Vet Res 1997; 58: 338-342.

20 Bannasch MJ and Foley JE. Epidemiologic evaluation of multiple respiratory pathogens in cats in animal shelters. J Feline Med Surg 2005; 7: 109-119.

21 Friedl Y, Schulz B, Knebl A, et al. Efficacy of passively transferred antibodies in cats with acute viral upper respiratory tract infection. Vet $J$ 2014; 201: 316-321.

22 Helps CR, Lait P, Damhuis A, et al. Factors associated with upper respiratory tract disease caused by feline herpesvirus, feline calicivirus, Chlamydophila felis and Bordetella bronchiseptica in cats: experience from 218 European catteries. Vet Rec 2005; 156: 669-673.

23 Veir JK, Ruch-Gallie R, Spindel ME, et al. Prevalence of selected infectious organisms and comparison of two anatomic sampling sites in shelter cats with upper respiratory tract disease. J Feline Med Surg 2008; 10: 551-557.

$24 \mathrm{Kahn}$ DE, Hoover EA and Bittle JL. Induction of immunity to feline caliciviral disease. Infect Immun 1975; 11: 1003-1009.

25 Di Martino B, Di Francesco CE, Meridiani I, et al. Etiological investigation of multiple respiratory infections in cats. New Microbiol 2007; 30: 455-461.

26 Marsilio F, Di Martino B, Decaro N, et al. A novel nested PCR for the diagnosis of calicivirus infections in the cat. Vet Microbiol 2005; 105: 1-7.

27 Huebner J. Meine Katze hustet! Neues zum Thema “Katzenschnupfen" [article in German]. Kleintierpraxis 2008; 53 : 390-392.

28 Harley R, Day S, Di Rocco C, et al. The Chlamydophila felis plasmid is highly conserved. Vet Microbiol 2010; 146: 172-174.

29 Marsilio F, Di Martino B and Di Francesco C. Use of a duplex-PCR assay to screen for Feline Herpesvirus-1 and Chlamydophila spp. in mucosal swabs from cats. New Microbiol 2004; 27: 287-292.

30 Binns SH, Dawson S, Speakman AJ, et al. A study of feline upper respiratory tract disease with reference to prevalence and risk factors for infection with feline calicivirus and feline herpesvirus. J Feline Med Surg 2000; 2: 123-133.

31 Gaston JZ, Stengel C, Harbour D, et al. Prävalenz des felinen Herpesvirus-1, felinen Calicivirus und von Chlamydophila felis in Mehrkatzenhaushalten [article in German]. Kleintierpraxis 2004; 33: 351-358.

32 Adler K, Radeloff I, Stephan B, et al. Bacteriological and virological status in upper respiratory tract infections of cats (cat common cold complex) [article in German]. Berl Munch Tierarztl Wochenschr 2007; 120: 120-125.

33 Low HC, Powell CC, Veir JK, et al. Prevalence of feline herpesvirus 1, Chlamydophila felis, and Mycoplasma spp DNA in conjunctival cells collected from cats with and without conjunctivitis. Am J Vet Res 2007; 68: 643-648.

34 Vogtlin A, Fraefel C, Albini S, et al. Quantification of feline herpesvirus 1 DNA in ocular fluid samples of clinically diseased cats by real-time TaqMan PCR. J Clin Microbiol 2002; 40:519-523.

35 Helps C, Reeves N, Egan K, et al. Detection of Chlamydophila felis and feline herpesvirus by multiplex realtime PCR analysis. J Clin Microbiol 2003; 41: 2734-2736.

36 Cai Y, Fukushi H, Koyasu S, et al. An etiological investigation of domestic cats with conjunctivitis and upper respiratory tract disease in Japan. J Vet Med Sci 2002; 64: 215-219. 
37 Burns RE, Wagner DC, Leutenegger CM, et al. Histologic and molecular correlation in shelter cats with acute upper respiratory infection. J Clin Microbiol 2011; 49: 2454-2460.

38 Knowles JO, McArdle F, Dawson S, et al. Studies on the role of feline calicivirus in chronic stomatitis in cats. Vet Microbiol 1991; 27: 205-219.

39 Dean E and Meunier V. Feline eosinophilic keratoconjunctivitis: a retrospective study of 45 cases (56 eyes). J Feline Med Surg 2013; 15: 661-668.
40 Hoover EA and Kahn DE. Experimentally induced feline calicivirus infection: clinical signs and lesions. J Am Vet Med Assoc 1975; 166: 463-468.

41 Gerriets W, Joy N, Huebner-Guthardt J, et al. Feline calicivirus: a neglected cause of feline ocular surface infections? Vet Ophthalmol 2012; 15: 172-179.

42 Sykes JE, Studdert VP, Anderson G, et al. Comparison of Chlamydia psittaci from cats with upper respiratory tract disease by polymerase chain reaction analysis of the ompA gene. Vet Rec 1997; 140: 310-313. 